\title{
Prenatal diagnosis of feta noncompaction cardiomyopathy with de novo CALM2 mutation
}

\author{
wen zhang ${ }^{1}$, xiaohui dai ${ }^{2}$, Hanmin $\mathrm{Liu}^{1}$, Lei $\mathrm{Li}^{1}$, Shu Zhou ${ }^{1}$, Qi Zhu ${ }^{2}$, and Jiao Chen ${ }^{1}$ \\ ${ }^{1}$ Affiliation not available \\ ${ }^{2}$ West China Second University Hospital,West China Medcical School,Sichuan Univeisity
}

August 9, 2021

\begin{abstract}
We report what apprears to be the first case of fetal noncompaction cardiomyopathy in both ventricles accompanied by a mutation in the calmodulin gene (CALM2): A 25-year-old woman was referred to our hospital at $25+1$ weeks of gestation for evaluation of fetal defects. A postnatal echocardiography showed biventricular noncompaction cardiomyopathy. After terminated the pregnancy, fetal noncompaction cardiomyopathy was comfirmed by autopsy and histopathologic examination. And the whole-exome sequencing of genomic DNA demonstrated a de novo heterozygous mutation (c.389A>G;p.D130G) in CALM2, whereas the parents were normal. In this case report, we highlight the gene mutation in noncompaction cardiomyopathy.
\end{abstract}

Prenatal diagnosis of feta noncompaction cardiomyopathy with de novo CALM 2 mutation: first description Wen Zhang ${ }^{1,2}$ \#, Xiaohui Dai ${ }^{1,2} \#$, Hanmin $\mathrm{Liu}^{2,3}$, Lei $\mathrm{Li}^{2,4}$, Shu Zhou ${ }^{2,5}$, Qi Zhu ${ }^{1,2}$ and Jiao Chen ${ }^{1,2 *}$

1. Department of Ultrasonic Medicine, West China Second University Hospital of Sichuan University, Chengdu, Sichuan 610041, China

2. Key Laboratory of Birth Defects and Related Diseases of Women and Children (Sichuan University), Ministry of Education, Chengdu, Sichuan 610041, China

3. Department of Pediatrics, West China Second University Hospital of Sichuan University, Chengdu, Sichuan 610041, China

4. Department of Pathology, West China Second University Hospital of Sichuan University, Chengdu, Sichuan 610041, China

5. Department of Obstetrics, West China Second University Hospital of Sichuan University, Chengdu, Sichuan 610041, China

\# These authors contributed equally to this work.

*Correspondence author, Tel/Fax: +86 28 85503744, Email:jiaochen2000@163.com

Abstract

We report what apprears to be the first case of fetal noncompaction cardiomyopathy in both ventricles accompanied by a mutation in the calmodulin gene (CALM2): A 25-year-old woman was referred to our hospital at $25^{+1}$ weeks of gestation for evaluation of fetal defects. A postnatal echocardiography showed biventricular noncompaction cardiomyopathy. After terminated the pregnancy, fetal noncompaction cardiomyopathy was comfirmed by autopsy and histopathologic examination. And the whole-exome sequencing of genomic DNA demonstrated a de novo heterozygous mutation (c.389A > G;p.D130G) in CALM2, whereas the parents were normal. In this case report, we highlight the gene mutation in noncompaction cardiomyopathy. 
Key words: Prenatal, Noncompaction cardiomyopathy, CALM2

\section{INTRODUCTION}

Noncompaction cardiomyopathy is a rare disorder that frequently manifests as monogenic diseases, especially neuromuscular disorders and chromosomal defects, and was first reported on autopsy in 1969 [1]. The incidence of noncompaction cardiomyopathy in the general population ranges from $0.05 \%$ to $0.25 \%$, whereas the incidence in children may reach $9.2 \%$ [2]. Noncompaction cardiomyopathy is characterized by increased numbers of prominent trabeculations and deep intertrabecular spaces. With the development of medical imaging techniques, the detection rate of noncompaction cardiomyopathy has increased, and the disorder may even be recognized as early as the fetal period. We herein present the first case of fetal noncompaction cardiomyopathy in both ventricles accompanied by a mutation in the calmodulin gene $(C A L M 2)$ at $25^{+1}$ weeks of gestation.

\section{CASE PRESENTATION}

A 25-year-old woman (gravida 1, para 0 ) was referred to our hospital at $25^{+1}$ weeks of gestation for evaluation of fetal defects. The patient was allergic to penicillin. Both parents were healthy, and there was no family history of birth defects or exposure to any specific teratogenic agents. A postnatal two-dimensional ultrasonographic investigation $(3.0-5.0 \mathrm{MHz})$ (Voluson E10; GE Healthcare, Chicago, IL, USA) showed biventricular noncompaction cardiomyopathy, slight pericardial effusion, and bradycardia (106 bpm). The ratio of noncompacted to compacted myocardium was about 3 in the left ventricle and about 2 in the right ventricle (Figure 1A). Color Doppler revealed blood perfusion to the intertrabecular recesses (Figure 1B). The cardiovascular profile score was 9 . Two weeks later, the fetal heart showed no significant improvement.

The couple decided to terminate the pregnancy by inducing labor with ethacridine lactate (Rivanol ${ }^{\circledR}$; Fengchen Group Co., Ltd., Qingdao, China). The woman vaginally delivered a stillborn child 2 days later. Genomic DNA was extracted from the muscle of the fetus to perform whole-exome sequencing. The result demonstrated a de novo heterozygous mutation (c.389A $>$ G;p.D130G) in CALM2(Figure 2), whereas the sequencing results of the parents were normal. At autopsy, the biventricular wall contained increased numbers of prominent trabeculae and deep intratrabecular recesses (Figure 3). Histopathologic examination confirmed fetal noncompaction cardiomyopathy (Figure 4).

\section{DISCUSSION}

Noncompaction is a rare cardiomyopathy with various genotypic and phenotypic manifestations. It is categorized as a primary genetic cardiomyopathy by the American Heart Association and as an unclassified cardiomyopathy by the European Society of Cardiology [3]. According to a study by Stöllberger et al. [4], the diagnostic criteria for noncompaction cardiomyopathy by echocardiography in pregnancy are as follows: in the end-diastolic stage, at least four trabeculations protruding apically to the papillary muscle of the left ventricle visible in one imaging plane; a two-layered structure with epicardial compacted and endocardial noncompacted layers and a noncompaction:compaction ratio of [?]2; and in color Doppler, intraventricular blood perfusing the intertrabecular spaces.

The disorder can be familial or sporadic and may be isolated or accompanied by other cardiac diseases. The etiology of noncompaction cardiomyopathy is complex and still unclear. Although at least 40 gene mutations are reportedly associated with noncompaction cardiomyopathy (e.g., $M Y H^{7} 7$ and PRDM16 [5-7]), few case reports of CALM2 mutation in fetal noncompaction cardiomyopathy have been published. CALM2 is a $\mathrm{Ca}^{2+}$-signaling gene that encodes for calmodulin and is associated with long QT syndrome (LQTS) phenotypes. In three reported cases, CALM2 mutation might have contributed to LQTS accompanied by cardiomyopathy (one case of hypertrophic cardiomyopathy and two cases of left ventricular noncompaction cardiomyopathy), indicating the variant positions in CALM2(c.396T >G;p.D132E, c.394G >C;p.D132H, and c395A > G;p.D132G) [8-10]. Our case is the first report of a novel CALM2 mutation (c.389A>G;p.D130G) in fetal noncompaction cardiomyopathy accompanied by bradycardia detected with whole-exome sequencing. Therefore, we highly suspect that CALM2 is associated with noncompaction cardiomyopathy, particularly in 
fetuses with bradycardia. Sinus bradycardia might be a manifestation of LQTS in the fetus [11]. Additionally, with reference to previously reported findings [8-10], we highly suspect that CALM2 variants might be associated with cardiomyopathy and arrhythmia, especially LQTS. Nonetheless, further research is required to confirm this hypothesis and elucidate the pathogenic mechanism.

\section{CONCLUSION}

With the improvement of the ultrasonic resolution, the detection rate of prenatal noncompaction cardiomyopathy was increased, and it is useful to recognize noncompaction cardiomyopathy as early as possible. Meanwhile, this case highlights the importance of genetic testing in the follow-up of prenatal disease, which will provide human databases with more information regarding gene defects, mutation sites, and various phenotypes.

\section{REFERENCES}

1. Finsterer J, C Stollberger, and JA Towbin, Left ventricular noncompaction cardiomyopathy: cardiac, neuromuscular, and genetic factors. Nat Rev Cardiol 2017; 14: 224-237.

2. Engberding R, et al, Isolated non-compaction cardiomyopathy. Dtsch Arztebl Int 2010; 107: 206-13.

3. Chebrolu LH, AM Mehta, and NC Nanda, Noncompaction cardiomyopathy: The role of advanced multimodality imaging techniques in diagnosis and assessment. Echocardiography 2017; 34: 279-289.

4. Stollberger C, C Wegner and J Finsterer, Fetal Ventricular Hypertrabeculation/Noncompaction: Clinical Presentation, Genetics, Associated Cardiac and Extracardiac Abnormalities and Outcome. Pediatr Cardiol 2015; 36: 1319-26.

5. Hoedemaekers YM, et al, Prenatal ultrasound diagnosis of MYH7 non-compaction cardiomyopathy. Ultrasound Obstet Gynecol 2013;41: 336-9.

6. Nomura Y, et al, A novel MYH7 gene mutation in a fetus with left ventricular noncompaction. Can J Cardiol 2015; 31:103 e1-3.

7. Delplancq G, et al, Cardiomyopathy due to PRDM16 mutation: First description of a fetal presentation, with possible modifier genes. Am J Med Genet C Semin Med Genet 2020; 184:129-135.

8. Zahavich L et al, Novel Association of a De Novo CALM2 Mutation With Long QT Syndrome and Hypertrophic Cardiomyopathy. Circ Genom Precis Med 2018; 11: 002255.

9. Pipilas DC, et al, Novel calmodulin mutations associated with congenital long QT syndrome affect calcium current in human cardiomyocytes. Heart Rhythm 2016; 13: 2012-9.

10. Makita N, et al, Novel calmodulin mutations associated with congenital arrhythmia susceptibility. Circ Cardiovasc Genet 2014; 7: 466-74.

11. Clur SB, et al, Left Ventricular Isovolumetric Relaxation Time Is Prolonged in Fetal Long-QT Syndrome. Circ Arrhythm Electrophysiol 2018; 11: 005797. 

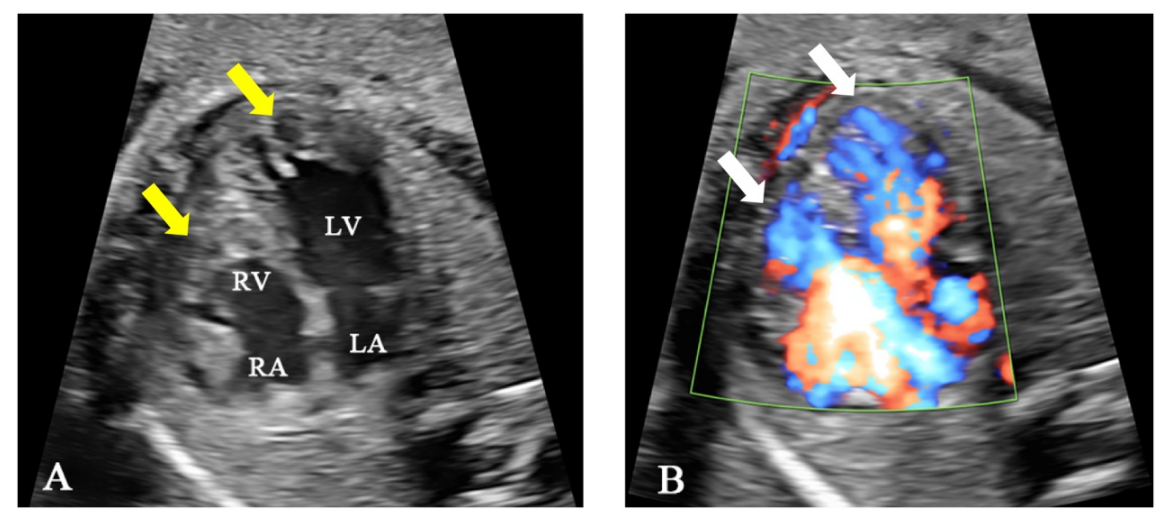

Figure 1. Fetal echocardiography at $25^{+1}$ weeks of gestation. (A) The two-dimensional ultrasound image shows increased numbers of prominent trabeculations and deep intertrabecular spaces in both ventricles (yellow arrow), especially at the left ventricular apex, with a noncompaction:compaction ratio of 3:1. (B) The color Doppler ultrasound image shows blood perfusing the intertrabecular recesses (white arrow). LA, left atrium; LV, left ventricle; RA, right atrium; RV, right ventricle.

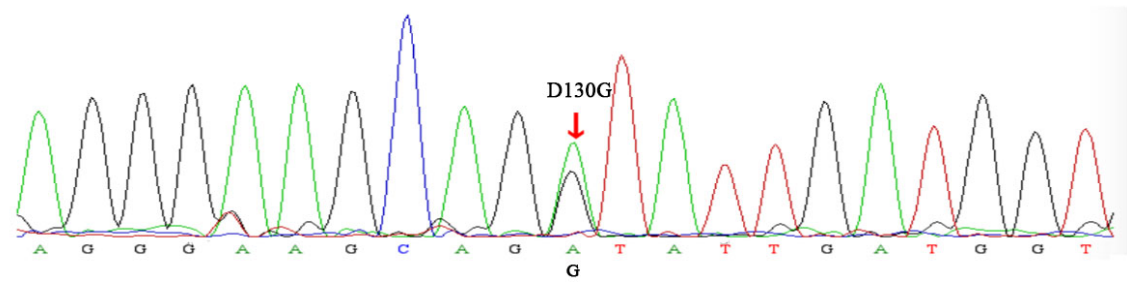

Figure 2. Sanger sequencing electropherogram. The variant (c.389A $>$ G) demonstrated the replacement of a conserved aspartic acid residue at position 130 with glycine (p.D130G) in the CALM2 gene (red arrow).

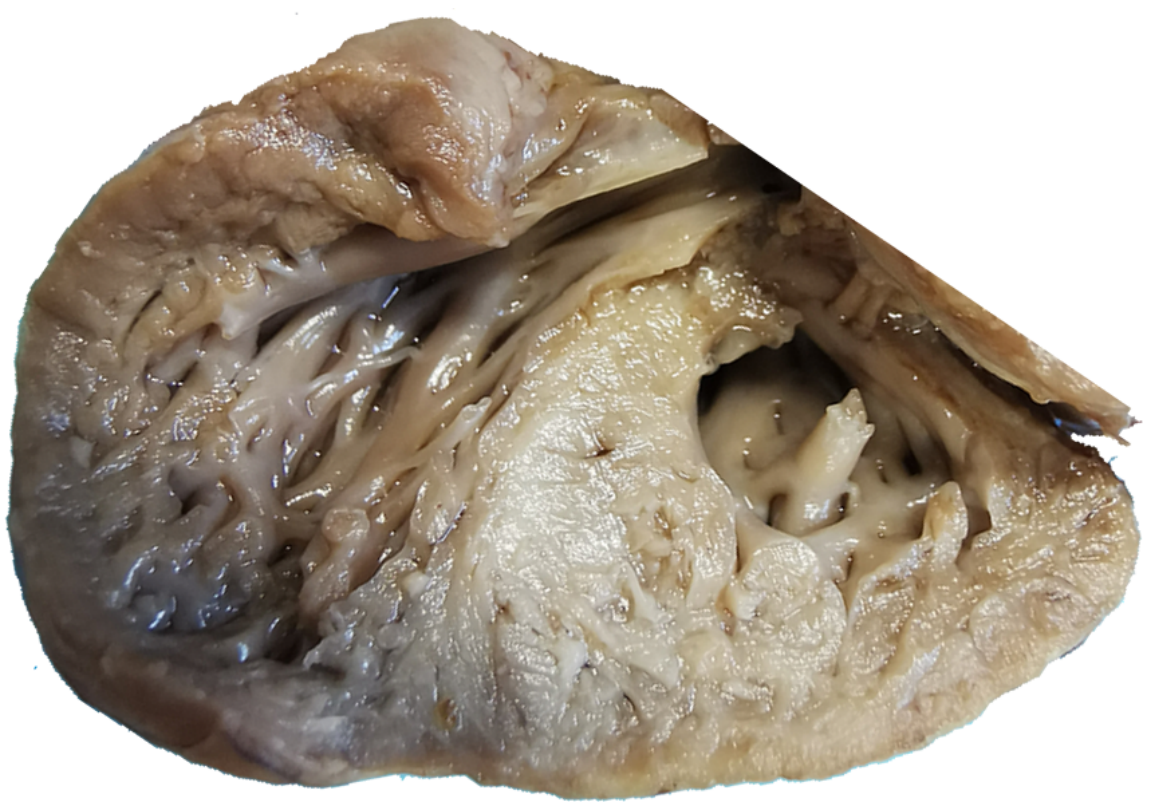


Figure 3. Dissected autopsy specimen. The specimen showed excessive trabeculae and deep intertrabecular recesses within the biventricular myocardium.

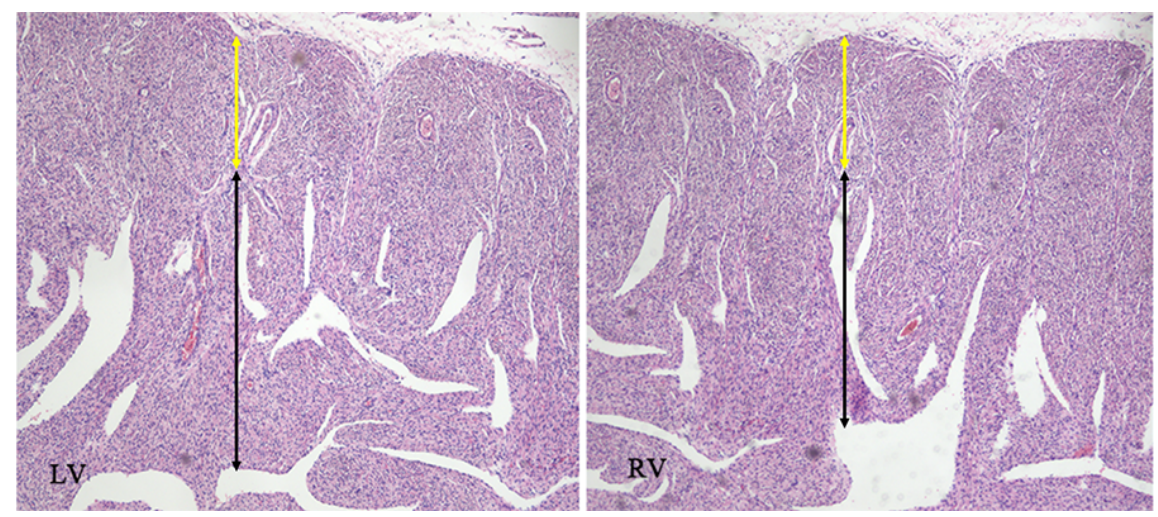

Figure 4. Histopathologic appearance of the myocardium at low magnification (hematoxylin and eosin, $\times 40)$. The images were compatible with noncompaction cardiomyopathy, with cardiomyocyte disarray in the noncompacted layer (black arrow) in opposition to regular cardiomyocytes in the compacted layer (yellow arrow).
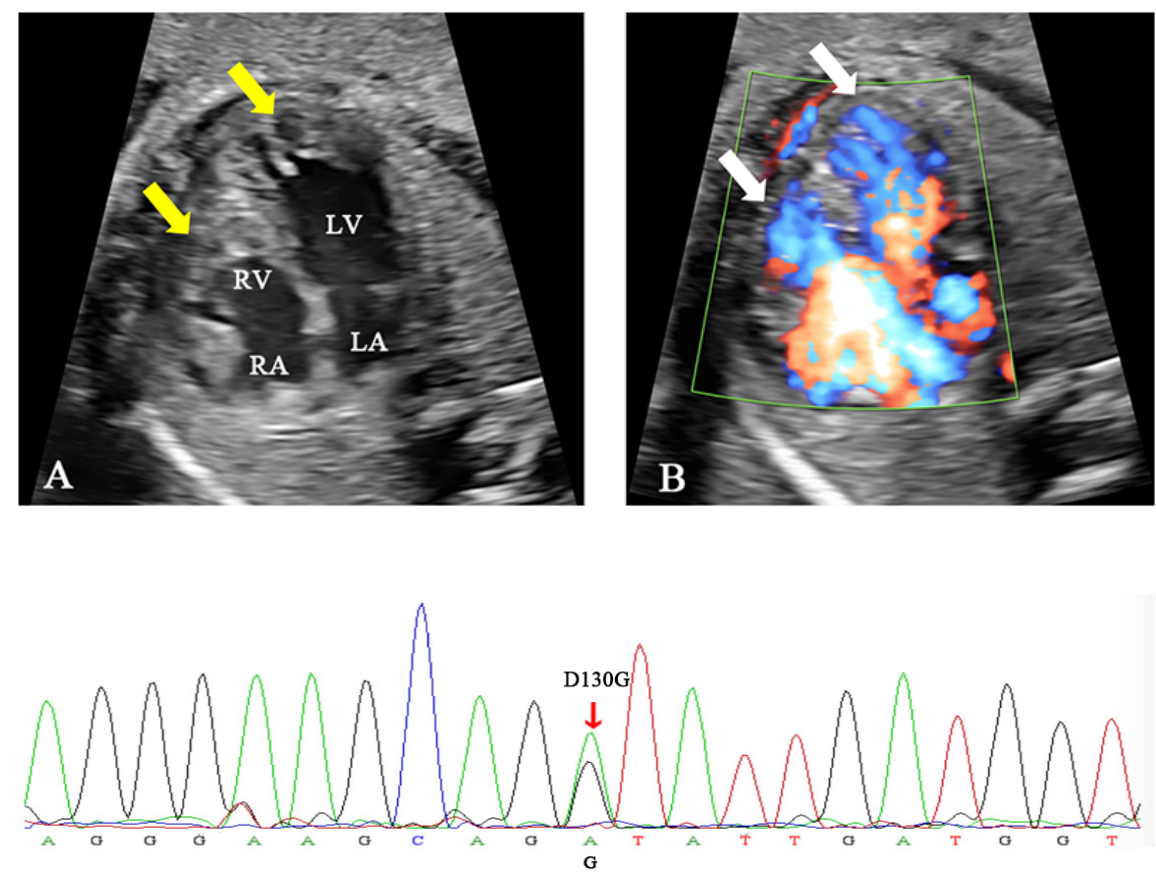

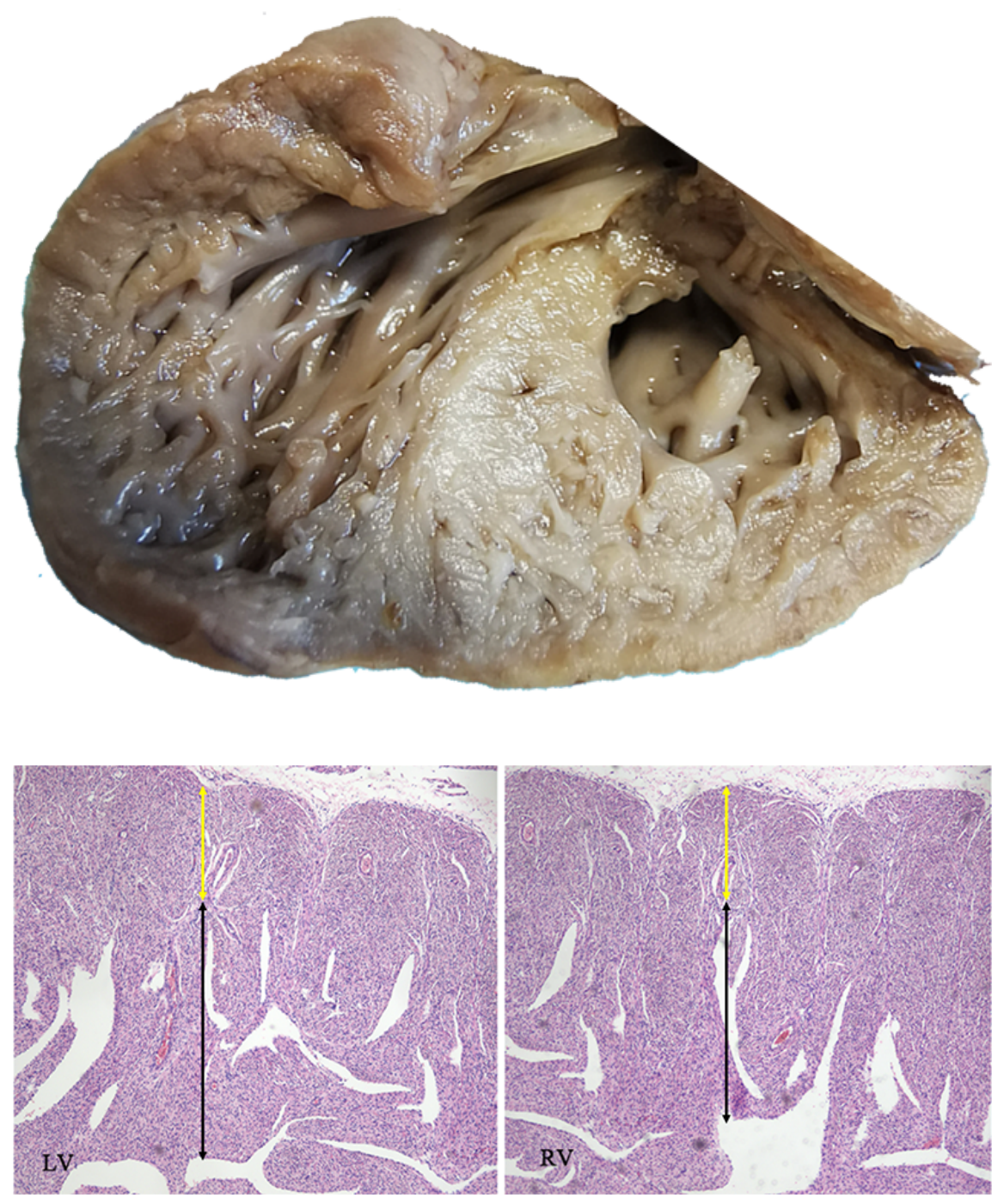